\title{
A OTIMIZAÇÃO DOS PESOS DAS OBSERVAÇÕES GEODÉSICAS POR UM PROBLEMA DE VALOR PRÓPRIO INVERSO: SOLUÇÃO PELO MÉTODO DE NEWTON E QUASE NEWTON - BFGS.
}

The optimization of geodetic observations weights through an eigenvalue problem: solution by the Newton and quase Newton - BFGS methods

\author{
QUINTINO DALMOLIN ${ }^{1}$ \\ REGINALDO DE OLIVEIRA ${ }^{2}$ \\ ${ }^{1}$ Universidade Federal do Paraná - SCT \\ Curso de Pós-Graduação em Ciências Geodésicas \\ Centro Politécnico - Jardim das Américas \\ Caixa Postal 19001 - CEP-81531 \\ E-mail: qdalmolin@ufpr.br \\ ${ }^{2}$ Universidade Tecnológica Federal do Paraná - UTFPR - \\ Coordenação de Mecânica - Campus Ponta Grossa \\ Av. Monteiro Lobato, Km 04 \\ CEP: 84.016-210 Ponta Grossa-PR \\ E-mail: reolivei@hotmail.com
}

\begin{abstract}
RESUMO
Neste trabalho apresenta-se a otimização dos pesos das observações baseada em um problema de valor próprio inverso e dois métodos para a sua solução. O método de Newton clássico (aproximação linear) e o método quase-Newton-BFGS (BroydenFletcher-Goldfarb-Shanno) que usa de modelos quadráticos para minimizar uma função. Apresentam-se ainda os fundamentos da otimização dos pesos das observações geodésicas formuladas através da análise dos valores próprios da matriz de covariâncias dos parâmetros estimados. Os métodos propostos são aplicados em três redes geodésicas bidimensionais locais e os resultados analisados e discutidos.
\end{abstract}

Palavras-chave: Otimização dos Pesos das Observações Geodésicas; Problema de Valor Próprio Inverso; Pré-Análise; Mínimos Quadrados. 


\begin{abstract}
In this paper, the optimization of geodetic observation weights through an eigenvalue problem and two methods for its solution are presented: the classic Newton method (linear estimate) and the quase-Newton - BFGS method which uses quadratic models to minimize a function. It's presented the basis for the optimization of the geodetic weighted observations formulated through the analysis of the eigenvalues in the covariance matrix estimated of the parameters. The proposed methods are applied in three local bi-dimensional geodetic networks and the results are analysed and discussed.
\end{abstract}

Keywords: Optimization of Geodetic Observations Weights; Eigenvalue Problem; Pre-Analysis; Least Squares.

\title{
1. INTRODUÇÃO
}

A idéia básica da otimização de redes geodésicas está no reconhecimento de que é possível estimar a sua qualidade antes de qualquer medição ser realizada. Em meio à comunidade que atua nas áreas das Ciências Geodésicas, é também conhecida como "Pré-Análise", a qual tem por objetivo projetar o levantamento geodésico de modo a obter resultados com a qualidade desejada, e com observações mais viáveis praticamente e economicamente. Em outras palavras poder-se-ia dizer que a Pré-Análise visa definir especificações ótimas, necessárias e suficientes, para o levantamento, de modo a produzir parâmetros com qualidade mínima desejada e pré-estabelecida (DALMOLIN, 2010 p.67).

Esta idéia abre a possibilidade de analisar detalhadamente o projeto da rede geodésica com respeito a sua precisão, confiabilidade, custos e ainda melhorar partes dela, se necessário. A precisão pode ser expressa em termos da matriz das covariâncias dos parâmetros estimados e representada geometricamente através da elipse dos erros (GEMAEL, 1994, p.234) . Conceitualmente, o trabalho do planejamento ótimo de uma rede geodésica é em geral, compreendido como a construção de uma configuração geométrica ótima e/ou de um plano de observações cujas realizações satisfaçam critérios de qualidade pré-definidos.

Já, GRAFAREND e SANSO (1985) classificaram os projetos de otimização de redes segundo as seguintes ordens: Projeto de Ordem Zero, quando se trata de escolher um sistema de referência (datum) ótimo; Projeto de Primeira Ordem, quando se trata de projetar uma configuração ótima para a rede; Projeto de Segunda Ordem, quando se deseja conhecer os pesos das observações que servirão de base para realização da rede (pesos otimizados) e; Projeto de Terceira Ordem, quando se deseja incluir novas observações para melhorar uma rede já existente. Nos casos em que projetos de primeira e de segunda ordem forem tratados simultaneamente é chamado de Projeto Combinado.

O problema de otimização de pesos é conhecido na literatura geodésica como planejamento ou projeto de segunda ordem, no qual se pretende uma precisão pré- 
definida para a rede e para a matriz dos coeficientes ou de configuração A, desing matrix na literatura Inglesa, cujos pesos são tratados como incógnitas.

A partir daí, pode-se obter pesos otimizados para as observações fazendo-se uso de modelos "ótimos" para a matriz de "critérios" ou de covariâncias dos parâmetros estimados. Desta forma, qualquer matriz definida positiva ou semidefinida positiva pode ser usada como uma matriz critério, desde que represente uma situação adequada para a precisão. Um caso particular de uma matriz critério homogênea e isotrópica de covariâncias é a matriz identidade, isto é, $\mathbf{Q}_{\mathbf{x}}=\mathbf{I}$.

Outra possibilidade para a otimização de pesos é usar valores próprios préestabelecidos. A qualidade do ajustamento é diagnosticada através dos valores e vetores próprios. Assim, pode-se formular a otimização dos pesos das observações geodésicas, por meio de condições impostas aos valores próprios (ou autovalores) da matriz de covariâncias $\mathbf{Q}_{\mathrm{x}}$, ou seja, os valores próprios da matriz de covariâncias se apresentam como função objetivo no processo de otimização.

A solução da formulação será obtida com o emprego do Problema de Valor Próprio Inverso iterativamente, aplicado sobre a matriz dos coeficientes do sistema das equações normais. Neste trabalho, o método de Newton clássico com aproximação linear apresentado por FRIEDLAND et al. (1987, p. 639-643) e o modelamento do problema por meio de uma função de minimização com modelos quadráticos serão usados para apresentar a solução e a análise da otimização dos pesos.

\section{FUNDAMENTOS TEÓRICOS}

A obtenção dos pesos otimizados para as observações pode ser realizada através do uso dos valores próprios associados à matriz de covariâncias dos parâmetros ajustados dada por,

$$
\mathbf{Q}_{\mathbf{x}}=\sigma_{0}^{2}\left(\mathbf{A}^{\mathrm{t}} \mathbf{P A}\right)^{+}=\sigma_{0}^{2} \mathbf{N}^{+}
$$

Onde, $\sigma_{0}^{2}$ é o fator de variância da unidade de peso que, em situações de planejamento de redes pode ser fixado como sendo a unidade. O sinal de "+" indica a inversa generalizada de Moore-Penrose, também conhecida como "pseudoinversa"; e $\mathbf{P}$ corresponde a matriz dos pesos dada pela inversa da matriz de covariâncias das observações, $\Sigma_{\ell_{\mathrm{b}}}$. Assim:

$$
\mathbf{P}=\sigma_{0}^{2} \Sigma_{\ell_{\mathrm{b}}}^{-1}
$$

Como os valores próprios da matriz $\mathbf{Q}_{\mathbf{x}}$ fornecem informações sobre a qualidade da rede, objetiva-se no processo de otimização de pesos, o 
estabelecimento de valores próprios que conduzam a matriz de covariâncias a apresentar uma estrutura espectral ideal. Neste processo de otimização, o valor próprio máximo da matriz $\mathbf{Q}_{\mathbf{x}}$ é de interesse particular, pois permite que se obtenha a precisão limite para quaisquer grandezas estimadas a partir da rede (NINKOV; SCHMITT, 1983, p. 217).

Com efeito, considerando o modelo matemático de cada equação de observação $f_{i}$, dentro do modelo paramétrico linearizado (GEMAEL, 1994, p.118) , tem-se:

$$
\mathbf{V}=\mathbf{A X}+\mathbf{L}
$$

Onde V representa o vetor dos resíduos; $a_{(i, j)}=\frac{\partial f_{(i, 1)}}{\partial X_{a_{(j, 1)}}}$ com $i=1,2, \ldots$ e j $=$ $1,2, \ldots \mathrm{u}$, sendo $\mathbf{n}$ o número de observações e u o número de parâmetros que representam os elementos da matriz planejamento $\mathbf{A} ; \mathbf{X}$ o vetor correção; $\mathbf{L}=\mathbf{L}_{0}-\mathbf{L}_{\mathrm{b}}$ sendo $\mathbf{L}_{0}$ função dos parâmetros aproximados $\mathrm{e} \mathbf{L}_{\mathrm{b}} \mathrm{o}$ vetor dos valores observados.

A lei de propagação de covariâncias (GEMAEL, 1994, p.120), conduz a seguinte expressão:

$$
\sigma_{f_{i}}^{2}=\sigma_{0}^{2} \mathbf{a}_{j}^{t} \mathbf{Q}_{x} \mathbf{a}_{j}
$$

sendo $\mathbf{a}_{\mathrm{j}}$ a j-ésima coluna da matriz $\mathbf{A}$.

Considerando o quociente Rayleigh (WANG; CHOW, 1994, p. 38) obtém-se uma estimativa para $\sigma_{\mathrm{f}}^{2}$ através da expressão,

$\mathbf{a}_{\mathrm{j}}^{\mathrm{t}} \mathbf{a}_{\mathrm{j}} \lambda_{\min } \leq \sigma_{\mathrm{f}}^{2} \leq \mathbf{a}_{\mathrm{j}}^{\mathrm{t}} \mathbf{a}_{\mathrm{j}} \lambda_{\max }$

$\operatorname{com} \lambda_{\max }$ e $\lambda_{\text {min }}$ obtidos da (1).

Da equação (6), decorre a exigência de que o valor próprio máximo, ou autovalor máximo, da matriz de covariâncias dos parâmetros estimados deve ser de magnitude mínima. Quanto maior for a magnitude do valor próprio máximo comparado com os demais valores próprios da matriz espectral, mais desfavorável e não homogêneo será o comportamento da precisão como mostrado graficamente na Figura 1 (DALMOLIN; OLIVEIRA, 2009, P.34). Nestas condições, o ideal seria que o espectro da matriz de covariâncias fosse o mais homogêneo possível. 
Figura 1 - Representação Gráfica do Espectro de uma Matriz.

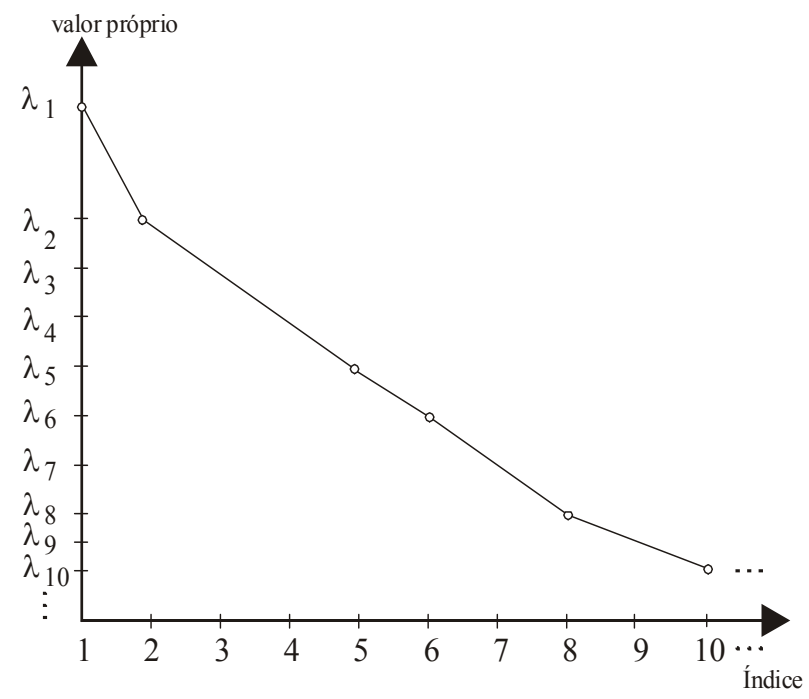

$\mathrm{Na}$ equação (1), conhecendo-se a geometria da rede representada pela matriz A, toda a influência sobre a magnitude dos valores próprios recai sobre a matriz dos pesos P. Como conseqüência, os valores próprios de (1) são dependentes da precisão das observações. Assim, conhecendo-se a matriz A, fixa-se os valores próprios para a equação (1) e toma-se a equação (3) como incógnita. Formula-se a otimização dos pesos das observações geodésicas usando o problema de valor próprio inverso para responder a seguinte pergunta:

Quais pesos (precisão para cada observação) devem ser atribuídos às observações para que a matriz $\mathbf{Q}_{\mathbf{x}}$, apresente um conjunto de valores próprios definidos antes da realização do levantamento em campo?

A resposta está em se estabelecer a ligação entre a otimização dos pesos das observações geodésicas e um problema de valor próprio inverso através da relação entre os valores próprios $\lambda_{\mathbf{i}}$ de $\mathbf{N}$ e $\mu_{\mathrm{i}}$ de $\mathbf{Q}_{\mathrm{x}}$ da seguinte forma:

$\lambda_{\mathrm{i}}=\frac{1}{\mu_{\mathrm{i}}}, \operatorname{com} \mu_{\mathrm{i}} \neq 0$ e $\mathrm{i}=1,2, \ldots$, u parâmetros, ,

uma vez que $\mathbf{N}$ e $\mathbf{Q}_{\mathbf{x}}$ apresentam valores próprios $\left(\lambda_{\mathrm{i}}\right.$ e $\left.\mu_{\mathrm{i}}\right)$ recíprocos entre si. Assim é possível formular a otimização dos pesos, relativa à matriz de coeficientes das incógnitas do sistema de equações normais N (BRONSON, 1993). 
Com base em (7) verifica-se que um aumento no valor próprio $\lambda_{\mathrm{i}}$ de $\mathbf{N}$ corresponde a um decréscimo no valor próprio $\mu_{\mathrm{i}}$ de $\mathbf{Q}_{\mathbf{x}}$, ou seja, para $\mu_{\mathrm{i}}$ tendendo para seu valor máximo, tem-se $\lambda_{\mathrm{i}}$ tendendo para seu valor mínimo. Todo o processo aplicado para a obtenção dos valores próprios pretendidos é efetuado sobre a matriz dos coeficientes das equações normais. Então, define-se o valor limite para a precisão dos parâmetros $\mu_{\max }$ e obtem-se os valores próprios para a matriz $\mathbf{N}$ através da relação (7).

\section{O PROBLEMA DE VALOR PRÓPRIO INVERSO APLICADO NA OTIMIZAÇÃO DOS PESOS DAS OBSERVAÇÕES GEODÉSICAS}

Para conceituar um problema de valor próprio inverso na otimização dos pesos, considera-se a matriz das equações normais obtida de $n$ observações e u parâmetros associada ao método dos mínimos quadrados na forma paramétrica.

Seja a matriz $\mathbf{N}(\mathbf{p})$, de ordem $u$, definida como,

$$
\mathbf{N}(\mathbf{p})=\sum_{\mathrm{j}=1}^{\mathrm{n}} \mathrm{p}_{\mathrm{j}} \mathbf{a}_{\mathrm{j}}^{\mathrm{t}} \mathbf{a}_{\mathrm{j}}
$$

onde o produto $\mathbf{a}_{\mathrm{j}}^{\mathrm{t}} \mathbf{a}_{\mathrm{j}}$ resulta numa matrizes real e simétrica de dimensão $\mathrm{u} \times \mathrm{u}$ e $\mathbf{p}=\left[\begin{array}{llllll}p_{1} & p_{2} & \cdots & p_{j} & \cdots & p_{n}\end{array}\right]^{t}$ um vetor que pertence ao espaço ndimensional dos números reais $\mathrm{R}^{\mathrm{n}}$. Denotando o conjunto dos $\mathrm{u}$ valores próprios de $\mathbf{N}(\mathbf{p})$ por $\lambda(\mathbf{p})=\left\{\lambda_{1}(\mathbf{p}), \lambda_{2}(\mathbf{p}), \ldots, \lambda_{\mathrm{i}}(\mathbf{p}), \ldots, \lambda_{\mathrm{u}}(\mathbf{p})\right\}, \quad$ com os elementos ordenados de forma crescente, $\lambda_{1}(\mathbf{p}) \leq \lambda_{2}(\mathbf{p}) \leq \ldots \leq \lambda_{\mathrm{i}}(\mathbf{p}) \leq \ldots \leq \lambda_{\mathrm{u}}(\mathbf{p})$ deve-se encontrar o vetor $\mathbf{p}$, $(\mathbf{p}=\operatorname{diag}(\mathbf{P}))$, que leva a matriz da $(8)$ à apresentar os valores próprios préestabelecidos $\quad \lambda^{*}=\left[\begin{array}{llllll}\lambda_{1}^{*} & \lambda_{2}^{*} & \ldots & \lambda_{\mathrm{i}}^{*} & \ldots & \lambda_{\mathrm{u}}^{*}\end{array}\right]^{\mathrm{t}}$ cujos elementos também estão em ordem crescente. Desta forma espera-se que para algum vetor $\mathbf{p}=\mathbf{p}^{*}$ se encontre a igualdade $\lambda_{i}\left(\mathbf{p}^{*}\right)-\lambda_{i}^{*}=\mathbf{0}$ ou $\operatorname{det}\left(\mathbf{N}(\mathbf{p})-\lambda_{i}^{*} \mathbf{I}\right)=0$ para $\mathrm{i}=1,2, \ldots, \mathrm{u}$. 


\subsection{Formulação do Problema de Valor Próprio Inverso}

Dados u números reais $\lambda_{1}^{*} \leq \lambda_{2}^{*} \leq \ldots \leq \lambda_{\mathrm{i}}^{*} \leq \ldots \leq \lambda_{\mathrm{u}}^{*}$, encontrar o vetor, $\mathbf{p} \in \mathrm{R}^{\mathrm{n}}$ tal que $\lambda_{\mathrm{i}}\left(\mathbf{p}^{*}\right)=\lambda_{\mathrm{i}}^{*}$ para $\mathrm{i}=1,2, \ldots, \mathrm{u}$.

Esta formulação pode ser colocada como um problema para solucionar um sistema de equações não-lineares, $\mathbf{F}(\mathbf{p})=\mathbf{0}$;

onde

$$
\mathbf{F}(\mathbf{p})=\left[\begin{array}{c}
\lambda_{1}(\mathbf{p})-\lambda_{1}^{*} \\
\vdots \\
\lambda_{\mathrm{i}}(\mathbf{p})-\lambda_{\mathrm{i}}^{*} \\
\vdots \\
\lambda_{\mathrm{u}}(\mathbf{p})-\lambda_{\mathrm{u}}^{*}
\end{array}\right]
$$

$\operatorname{com} \lambda_{1}(\mathbf{p}) \leq \lambda_{2}(\mathbf{p}) \leq \ldots \leq \lambda_{\mathrm{i}}(\mathbf{p}) \leq \ldots \leq \lambda_{\mathrm{u}}(\mathbf{p})$

Um algoritmo para a solução do sistema não linear (9) é o método de Newton clássico (puro), que para o caso de otimização dos pesos apresenta os seguintes passos:

Para $\mathrm{k}=0,1,2, \ldots$ segue-se,

1) Cálculo de $\mathbf{N}\left(\mathbf{p}^{\mathrm{k}}\right), \lambda_{\mathrm{i}}\left(\mathbf{p}^{\mathrm{k}}\right)$ e $\mathbf{m}_{\mathrm{i}}\left(\mathbf{p}^{\mathrm{k}}\right)$ para $\mathrm{i}=1,2, \ldots, \mathrm{u}$;

2) Calcular a norma $\left\|\mathbf{F}\left(\mathbf{p}^{k}\right)\right\|$

3) Parar se $\left\|\mathbf{F}\left(\mathbf{p}^{\mathrm{k}}\right)\right\| \leq \varepsilon$, onde $\varepsilon$ representa a qualidade da aproximação, ou seja, um critério de parada estabelecido. Por exemplo, $\varepsilon=10^{-8}$ e $\|(\bullet)\|$ representa a norma de um vetor;

4) Calcular $\mathbf{J}\left(\mathbf{p}^{\mathrm{k}}\right)$ e $\mathbf{F}\left(\mathbf{p}^{\mathrm{k}}\right)$ e obter $\mathbf{s}_{\mathrm{k}}$ solucionando o sistema $\mathbf{J}\left(\mathbf{p}^{\mathrm{k}}\right)_{\mathrm{k}}=-\mathbf{F}\left(\mathbf{p}^{\mathrm{k}}\right)$;

5) Calcular $\mathbf{p}^{\mathrm{k}+1}=\mathbf{p}^{\mathrm{k}}+\mathbf{s}_{\mathrm{k}}$;

6) Fazer $\mathrm{k}=\mathrm{k}+1$ e voltar ao passo 1 . 
Nas relações acima tem-se a chamada matriz Jacobiana, cuja expressão matemática é dada por,

$$
\mathbf{J}(\mathbf{p})=\left[\begin{array}{cccc}
\frac{\partial \mathrm{F}_{1}(\mathbf{p})}{\partial \mathrm{p}_{1}} & \frac{\partial \mathrm{F}_{1}(\mathbf{p})}{\partial \mathrm{p}_{2}} & \cdots & \frac{\partial \mathrm{F}_{1}(\mathbf{p})}{\partial \mathrm{p}_{\mathrm{n}}} \\
\frac{\partial \mathrm{F}_{2}(\mathbf{p})}{\partial \mathrm{p}_{1}} & \frac{\partial \mathrm{F}_{2}(\mathbf{p})}{\partial \mathrm{p}_{2}} & \cdots & \frac{\partial \mathrm{F}_{2}(\mathbf{p})}{\partial \mathrm{p}_{\mathrm{n}}} \\
\vdots & \vdots & \vdots & \vdots \\
\frac{\partial \mathrm{F}_{\mathrm{u}}(\mathbf{p})}{\partial \mathrm{p}_{1}} & \frac{\partial \mathrm{F}_{\mathrm{u}}(\mathbf{p})}{\partial \mathrm{p}_{2}} & \cdots & \frac{\partial \mathrm{F}_{\mathrm{u}}(\mathbf{p})}{\partial \mathrm{p}_{\mathrm{n}}}
\end{array}\right] .
$$

A matriz das derivadas segundas, necessárias para o cálculo da matriz Hessiana, como será visto a diante, é expressa matematicamente por,

$$
\nabla^{2} \mathrm{~F}=\frac{\partial^{2} \mathrm{~F}}{\partial \mathrm{p}_{\mathrm{i}} \partial \mathrm{p}_{\mathrm{j}}} \mathrm{i}, \mathrm{j}=1,2, \ldots, \mathrm{n}
$$

Para obter as grandezas como definidas acima, faz-se necessário a primeira e a segunda derivada do i-ésimo valor próprio $\lambda_{i}(\mathbf{p})$, cujas expressões são respectivamente (OLIVEIRA, 2003, pp. 42-46)

$$
\frac{\partial \lambda_{i}}{\partial \mathbf{p}_{\mathrm{k}}}=\left(\mathbf{m}_{\mathrm{i}}(\mathbf{p})\right)^{\mathrm{t}} \frac{\partial \mathbf{N}(\mathbf{p})}{\partial \mathrm{p}_{\mathrm{k}}} \mathbf{m}_{\mathrm{i}}(\mathbf{p})
$$

$\mathrm{e}$

$$
\frac{\partial \lambda_{i}}{\partial p_{k} \partial p_{p}}=\lambda_{i, k, p}=2 \sum_{\substack{j=1 \\ j \neq i}}^{n}\left(\frac{\left(\left(\mathbf{m}_{j}(\mathbf{p})\right)^{t} \frac{\partial \mathbf{N}(\mathbf{p})}{\partial p_{p}} \mathbf{m}_{i}(\mathbf{p})\right)\left(\left(\mathbf{m}_{j}(\mathbf{p})\right)^{\mathrm{t}} \frac{\partial \mathbf{N}(\mathbf{p})}{\partial p_{k}} \mathbf{m}_{i}(\mathbf{p})\right)}{\lambda_{i}(\mathbf{p})-\lambda_{j}(\mathbf{p})}\right)
$$

$\operatorname{para} \lambda_{\mathrm{i}} \neq \lambda_{\mathrm{j}}$.

Nas expressões acima, é importante notar que $\mathbf{m}_{\mathbf{i}}(\mathbf{p})$ é o i-ésimo vetor próprio obtido da matriz (8) que é função do valor atual atribuído à matriz dos 
pesos. Ainda, $\frac{\partial \mathbf{N}(\mathbf{p})}{\partial \mathrm{p}_{\mathrm{k}}}=\mathbf{a}_{\mathrm{k}}^{\mathrm{t}} \mathbf{a}_{\mathrm{k}}$ é derivada da equação (8) em relação ao k-ésimo peso sendo $\mathbf{a}_{\mathrm{k}}$ a k-ésima linha da matriz planejamento A.

Uma segunda maneira de solucionar um problema de valor próprio inverso é a utilização do Método Quase Newton - BFGS. Neste caso, pode-se formular uma função de minimização construída com a equação (9) da seguinte forma:

$$
\min _{\mathbf{p} \in \mathrm{R}^{\mathrm{n}}} \mathrm{f}(\mathbf{p}): \frac{1}{2} \mathbf{F}(\mathbf{p})^{\mathrm{t}} \mathbf{F}(\mathbf{p})
$$

Assim, verifica-se que uma solução para (14) coincide com uma solução para (9). Desta forma pode-se procurar uma solução para a equação acima utilizando modelos quadráticos (DENIS JR E SCHNABEL, 1993).

A aproximação de $\mathrm{f}$ dada na (14) em um ponto $\mathbf{p}^{\mathrm{k}}$, por meio de uma função quadrática, utilizando a expansão de Taylor truncada na ordem 2, é representado por,

$\mathrm{m}_{\mathrm{k}}(\mathbf{p})=\mathrm{f}\left(\mathbf{p}^{\mathrm{k}}\right)+\nabla \mathrm{f}\left(\mathbf{p}^{\mathrm{k}}\right)\left(\mathbf{p}-\mathbf{p}^{\mathrm{k}}\right)+\frac{1}{2}\left(\mathbf{p}-\mathbf{p}^{\mathrm{k}}\right) \nabla^{2} \mathrm{f}\left(\mathbf{p}^{\mathrm{k}}\right)\left(\mathbf{p}-\mathbf{p}^{\mathrm{k}}\right)^{2}$

onde, $\mathrm{m}_{\mathrm{k}}(\mathbf{p})$ representa o modelo quadrático corrente ou modelo quadrático obtido no k-ésimo passo do processo iterativo;

$\mathrm{f}\left(\mathbf{p}^{\mathrm{k}}\right)=\frac{1}{2} \mathbf{F}\left(\mathbf{p}^{\mathrm{k}}\right)^{\mathrm{t}} \mathbf{F}\left(\mathbf{p}^{\mathrm{k}}\right)$ é o valor numérico da função (14) no ponto $\mathbf{p}^{\mathrm{k}}$; $\nabla \mathrm{f}\left(\mathbf{p}^{\mathrm{k}}\right)=\mathbf{J}\left(\mathbf{p}^{\mathrm{k}}\right)^{\mathrm{t}} \mathbf{F}\left(\mathbf{p}^{\mathrm{k}}\right)$ corresponde ao gradiente da função $\mathrm{f}\left(\mathbf{p}^{\mathrm{k}}\right)$ no ponto $\mathbf{p}^{\mathrm{k}}$ e; $\nabla^{2} \mathrm{f}\left(\mathbf{p}^{\mathrm{k}}\right)=\mathbf{J}\left(\mathbf{p}^{\mathrm{k}}\right)^{\mathrm{t}} \mathbf{J}\left(\mathbf{p}^{\mathrm{k}}\right)+\mathbf{S}\left(\mathbf{p}^{\mathrm{k}}\right)$ à segunda derivada ou matriz Hessiana da função (14) no ponto $\mathbf{p}^{\mathrm{k}}$, com

$\mathbf{S}\left(\mathbf{p}^{\mathrm{k}}\right)=\sum_{\mathrm{j}=1}^{\mathrm{m}}\left(\mathrm{F}_{\mathrm{j}}\left(\mathbf{p}^{\mathrm{k}}\right) \nabla^{2} \mathrm{~F}_{\mathrm{j}}\left(\mathbf{p}^{\mathrm{k}}\right)\right)$

que apresenta as informações sobre a segunda derivada da função $\mathbf{F}(\mathbf{p})$ no ponto $\mathbf{p}^{\mathrm{k}}$, como apresentado em (JÄGER e KALTENBACH, 1990).

Utilizando modelos quadráticos, um ponto crítico ou de sela de mínimo (ou máximo) também é definido como o ponto $\mathbf{p}^{+}$onde $\nabla \mathrm{m}_{\mathrm{k}}\left(\mathbf{p}^{+}\right)=0$. Ou seja, 
onde a função gradiente se anula. Se o ponto de mínimo é procurado, deve-se ter, adicionalmente, a matriz Hessiana, positiva semidefinida. Ou seja, $\nabla^{2} \mathrm{f}\left(\mathbf{p}^{\mathrm{k}}\right) \geq 0$ (NOCEDAL E WRIGHT, 1999, p. 16).

A implementação do Método de Newton para minimizar a função (14), exige a resolução do sistema linear dado por,

$\left[\mathbf{J}\left(\mathbf{p}^{\mathrm{k}}\right)^{\mathrm{t}} \mathbf{J}\left(\mathbf{p}^{\mathrm{k}}\right)+\mathbf{S}\left(\mathbf{p}^{\mathrm{k}}\right)\right] \mathbf{s}_{\mathbf{k}}=-\nabla \mathrm{f}\left(\mathbf{p}^{\mathrm{k}}\right)$

cuja solução para cada iteração é representado por,

$$
\mathbf{s}_{\mathrm{k}}=-\left(\nabla^{2} \mathbf{F}\left(\mathbf{p}^{\mathrm{k}}\right)\right)^{-1} \nabla \mathrm{f}\left(\mathbf{p}^{\mathbf{k}}\right)
$$

A idéia é construir aproximações razoáveis da matriz Hessiana, representadas por $\mathbf{B}_{\mathrm{k}}$, em vez de escrevê-la explicitamente, em cada passo $\mathrm{k}$ do processo iterativo ou então por aproximações da sua inversa representada por $\mathbf{H}_{\mathrm{k}}$, ou seja, $\mathbf{H}_{\mathrm{k}}$ que corresponde a $\mathbf{B}_{\mathrm{k}}^{-1}$.

Reescrevendo a solução de (18) com essa consideração tem-se

$$
\mathrm{s}_{\mathrm{k}}=-\left(\mathbf{B}_{\mathrm{k}}\right)^{-1} \nabla \mathrm{f}\left(\mathbf{p}^{\mathrm{k}}\right)
$$

ou

$$
\mathrm{s}_{\mathrm{k}}=-\mathbf{H}_{\mathrm{k}} \nabla \mathrm{f}\left(\mathbf{p}^{\mathrm{k}}\right)
$$

O algoritmo que pode ser usado nas condições acima é o Algoritmo BFGS Globalizado, enunciado como segue (MARTINEZ e SANTOS, 1995, p.117):

$$
\text { Dados } \alpha \in(0,1), \beta>0, \theta \in(0,1), \quad \mathbf{p}^{0} \in \mathrm{R}^{\mathrm{n}}, \mathbf{H}_{0}=\mathbf{H}_{0}^{\mathrm{t}},
$$

$\mathbf{H}_{0}>0,\left(\right.$ por exemplo, $\left.\mathbf{H}_{0}=\mathbf{I}\right)$, com $\alpha, \beta$ e $\theta$ arbitrados nos intervalos acima.

$$
\text { Dados } \mathbf{p}^{\mathrm{k}} \text { e } \mathbf{H}_{\mathrm{k}} \text { faz-se: }
$$

1. $\operatorname{Se} \nabla \mathrm{f}\left(\mathbf{p}^{\mathrm{k}}\right)=0$ parar. Caso contrário ir para o passo 2.

$$
\text { 2. Estimar } \mathbf{s}_{\mathrm{k}}=-\mathbf{H}_{\mathrm{k}} \nabla \mathrm{f}\left(\mathbf{p}^{\mathrm{k}}\right) \text {; }
$$




$$
\begin{aligned}
& \text { 3. Se } \nabla \mathrm{f}\left(\mathbf{p}^{\mathrm{k}}\right)_{\mathbf{s}_{\mathrm{k}}}>-\theta\left\|\nabla \mathrm{f}\left(\mathbf{p}^{\mathrm{k}}\right)\right\| \mathbf{s}_{\mathrm{k}} \| \text {, faz-se } \mathbf{s}_{\mathrm{k}}=-\nabla \mathrm{f}\left(\mathbf{p}^{\mathrm{k}}\right) \text { e } \mathbf{H}_{\mathrm{k}}=\mathbf{I} \text {. } \\
& \text { Se }\left\|\mathbf{s}_{\mathrm{k}}\right\|<\beta\left\|\nabla \mathrm{f}\left(\mathbf{p}^{\mathrm{k}}\right)\right\| \text {, deve-se substituir } \mathbf{s}_{\mathrm{k}} \text { por } \frac{\beta \boldsymbol{f}\left(\mathbf{p}^{\mathrm{k}}\right) \| \mathbf{s}_{\mathrm{k}}}{\left\|\mathbf{s}_{\mathrm{k}}\right\|} ;
\end{aligned}
$$

4. Escolhe-se t que satisfaça a condição de Armijo:

$$
\begin{aligned}
& \begin{aligned}
& \mathrm{f}\left(\mathbf{p}^{\mathrm{k}}+\mathrm{ts}_{\mathrm{k}}\right) \leq \mathrm{f}\left(\mathbf{p}^{\mathrm{k}}\right)+ \operatorname{t\alpha } \nabla \mathrm{f}\left(\mathbf{p}^{\mathrm{k}}\right)^{\mathrm{t}} \mathbf{s}_{\mathrm{k}}, \text { onde t sobrescrito re } \\
& \text { transposição e; } \\
& \text { 5. Define-se } \mathbf{p}^{\mathrm{k}+1}=\mathbf{p}^{\mathrm{k}}+\mathrm{t} \mathbf{p}^{\mathrm{k}}, \mathbf{s}_{\mathrm{k}}=\mathbf{p}^{\mathrm{k}+1}-\mathbf{p}^{\mathrm{k}},
\end{aligned} \\
& \mathbf{y}_{\mathrm{k}}=\nabla \mathrm{f}\left(\mathbf{p}^{\mathrm{k}+1}\right)-\nabla \mathrm{f}\left(\mathbf{p}^{\mathrm{k}}\right),
\end{aligned}
$$$$
\text { Se } \mathbf{s}_{\mathrm{k}}^{\mathrm{t}} \mathbf{y}_{\mathrm{k}} \leq 0 \text { então } \mathbf{H}_{\mathrm{k}+1}=\mathbf{H}_{\mathrm{k}} \text {. Caso contrário, }
$$$$
\mathbf{H}_{k+1}=\mathbf{H}_{k}+\frac{\left(\mathbf{s}_{k}-\mathbf{H}_{k} \mathbf{y}_{k}\right) \mathbf{s}_{k}^{t}+\mathbf{s}_{k}\left(\mathbf{s}_{k}-\mathbf{H}_{k} \mathbf{y}_{k}\right)^{t}}{\mathbf{s}_{k} \mathbf{y}_{k}^{t}}-\frac{\left(\mathbf{s}_{k}-\mathbf{H}_{k} \mathbf{y}_{k}\right)^{t} \mathbf{y}_{k} \mathbf{s}_{k} \mathbf{s}_{k}^{t}}{\left(\mathbf{s}_{k}^{t} \mathbf{y}_{k}\right)^{2}}
$$

6. Fazer $\mathrm{k}=\mathrm{k}+1$ e voltar ao passo 1 .

O algoritmo BFGS tem a propriedade de produzir, em geral, matrizes definidas positivas e, portanto, com direções de descida, que freqüentemente não precisarão de correção. Isto é, na estratégia de busca linear, o algoritmo gera uma quantidade de tentativas para buscar, ao longo desta direção o ponto, cujo valor proporcione decréscimo da função. Neste novo ponto, uma nova direção de busca é obtida e também um novo valor para t é calculado (MARTINEZ; SANTOS, 1995, p. 116).

No passo 3 usou-se $\beta=\frac{1}{\left\|\mathbf{H}_{\mathrm{k}}^{-1}\right\|}$ adaptando-se aqui as informações de

MARTINEZ e SANTOS (1995). Na condição do passo 4, conhecida como condição de Armijo, escolhe-se inicialmente $t=1$ e quando esta condição falha adota-se de forma iterativa $\mathrm{t}=0,5 \overline{\mathrm{t}}$, onde $\overline{\mathrm{t}}$ é o último valor que fracassou até que a condição seja satisfeita. Implementações mais eficientes para obter o valor de 
t podem ser encontradas em Dennis JR. e Schnabel (1983) e Nocedal e Wright (1999).

Especificamente para este caso aplica-se o algoritmo BFGS para evitar a formação explicita da matriz Hessiana cuja formação necessita da equação (13) e desta forma contornar os casos em que algum passo do processo iterativo se tenha $\lambda_{i}(\mathbf{p})-\lambda_{j}(\mathbf{p})=0$ ou aproximadamente igual a zero, para $\mathrm{i} \neq \mathrm{j}$.

A seguir apresentam-se três experimentos onde os dois métodos propostos são aplicados para a obtenção dos pesos das observações.

\section{EXPERIMENTOS}

Os experimentos realizados para aplicação dos métodos aqui estudados referem-se a redes geodésicas bidimensionais. Pretende-se projetar as redes para que atendam a precisões pré-definidas. Neste caso, os pesos devem ser obtidos, pois, considera-se que as geometrias das redes são conhecidas, ou seja, fez-se o reconhecimento do levantamento em campo sobre o tipo de observações que serão feitas. Desta forma, os pesos devem ser obtidos no processo de otimização para que se possa selecionar a instrumentação compatível com a precisão final esperada.

Após a obtenção dos pesos no processo de otimização, a precisão necessária para cada observação pode ser obtida diretamente pela aplicação da equação (3).

\subsection{Experimento 1}

Neste experimento, estuda-se a obtenção dos pesos das observações geodésicas utilizando o método de Newton clássico e o quase Newton com o algoritmo BFGS. O problema de valor próprio inverso será aplicado para o planejamento e ajustamento das coordenadas cartesianas de um ponto a partir de quatro observações (duas de distância e duas de direção) como representado na Figura 2.

Figura 2 - Representação Geométrica do Planejamento de um Ponto em

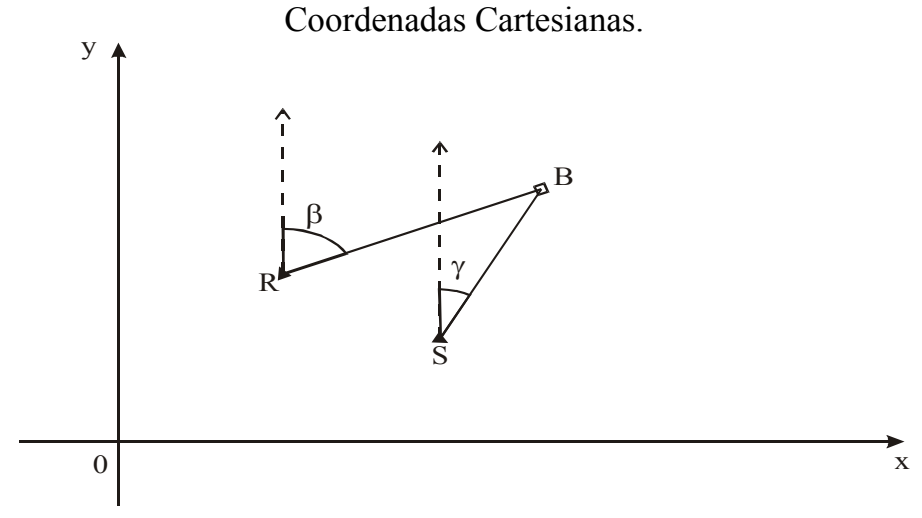

Bol. Ciênc. Geod., sec. Artigos, Curitiba, v. 16, n 4, p.538-556, out-dez, 2010. 
A representação geométrica do problema, como apresentada na Figura 2, estabelece que as observações planejadas serão constituídas de duas medidas de distância e duas de direção. O critério de parada para ambos os casos será $\varepsilon=10^{-8}$.

As coordenadas conhecidas dos pontos $\mathrm{R}$ e $\mathrm{S}$ são apresentados na Tabela 1, e tomou-se como coordenadas aproximadas do ponto B, $600 \mathrm{~m}$ e $582 \mathrm{~m}$ respectivamente para $\mathrm{x}$ e $\mathrm{y}$.

Tabela 1 - Coordenadas Horizontais.

\begin{tabular}{c|c|c}
\hline Ponto & $\mathrm{x}(\mathrm{m})$ & $\mathrm{y}(\mathrm{m})$ \\
\hline $\mathrm{R}$ & 200 & 500 \\
\hline $\mathrm{S}$ & 400 & 300 \\
\hline
\end{tabular}

Os valores próprios pré-estabelecidos para a matriz $\mathbf{N}$ foram: $\lambda^{*}=10000 \mathrm{e}$ $\lambda^{*}=8000$. Desta forma, o valor limite para a variância de qualquer parâmetro 2

estimado desta rede será de $\frac{1}{8000} \mathrm{~m}^{2}=0,000125 \mathrm{~m}^{2}$, que é o valor próprio máximo estabelecido para a matriz de covariâncias.

Para este caso, ambos o métodos obtiveram uma solução apresentada no Quadro 1.

Quadro 1 - Resultado da Otimização para o Experimento 1.

\begin{tabular}{|c|c|c|c|}
\hline \multicolumn{2}{|c|}{ Método } & Quase Newton-BFGS & Newton Clássico \\
\hline \multirow{4}{*}{ Pesos } & $\mathrm{D}_{\mathrm{BR}}$ & 3959,445618 & 3959,459555 \\
\cline { 2 - 4 } & $\mathrm{D}_{\mathrm{BS}}$ & 6409,439811 & 6409,425146 \\
\cline { 2 - 4 } & $\beta$ & 431907992,492078 & 431907687,076524 \\
\cline { 2 - 4 } & $\gamma$ & 602467427,629097 & 602467932,553265 \\
\hline
\end{tabular}

Ambos os métodos produziram um conjunto de pesos que ao se ajustar as coordenadas do ponto apresentou a mesma solução. Os dois primeiros pesos do quadro acima se referem às observações de distância e os dois últimos às observações de direção.

\subsection{Experimento 2}

No exemplo a seguir formula-se um problema de valor próprio inverso aplicado à Lei de Propagação de Covariâncias utilizada após um ajustamento por mínimos quadrados na sua forma paramétrica. 
$\mathrm{Na}$ Figura 3 representa-se uma rede geodésica bidimensional onde as coordenadas dos pontos $\mathrm{P}\left(\mathrm{x}_{\mathrm{p}}, \mathrm{y}_{\mathrm{p}}\right) \mathrm{e} \mathrm{Q}\left(\mathrm{x}_{\mathrm{q}}, \mathrm{y}_{\mathrm{q}}\right)$ são conhecidas e consideradas isentas de erros. As observações são as distâncias entre os pontos e os azimutes em $\mathrm{P}$ e Q contados a partir no Norte.

Figura 3 - Rede Geodésica Bidimensional.

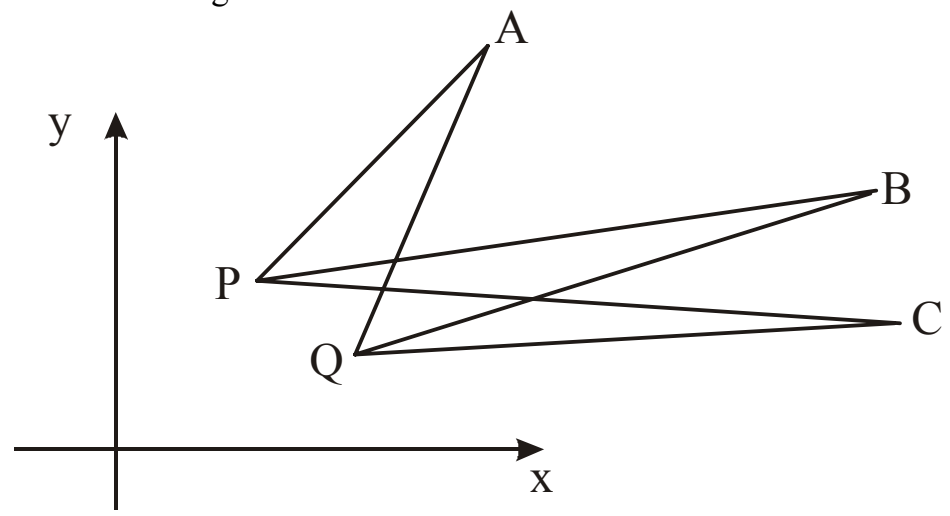

As coordenadas dos pontos $\mathrm{A}\left(\mathrm{x}_{\mathrm{a}}, \mathrm{y}_{\mathrm{a}}\right), \mathrm{B}\left(\mathrm{x}_{\mathrm{b}}, \mathrm{y}_{\mathrm{b}}\right)$ e $\mathrm{C}\left(\mathrm{x}_{\mathrm{c}}, \mathrm{y}_{\mathrm{c}}\right)$, designadas como parâmetros, devem ser estimadas com uma precisão pré-definida.

As coordenadas dos pontos fixos e também as coordenadas aproximadas dos parâmetros são apresentadas na Tabela 2 .

Tabela 2 - Coordenadas Horizontais de uma Rede Geodésica Bidimensional.

\begin{tabular}{c|c|c}
\hline \multirow{2}{*}{ pontos } & \multicolumn{2}{|c}{ Coordenadas dos Pontos Fixos } \\
\cline { 2 - 3 } & $\mathrm{x}(\mathrm{m})$ & $\mathrm{y}(\mathrm{m})$ \\
\hline $\mathrm{P}$ & 2,1 & 2,5 \\
\hline $\mathrm{Q}$ & 3,5 & 1,3 \\
\hline \multirow{2}{*}{ pontos } & \multicolumn{2}{|c}{ VALORES APROXIMADOS } \\
\cline { 2 - 3 } & $\mathrm{x}(\mathrm{m})$ & $\mathrm{y}(\mathrm{m})$ \\
\hline $\mathrm{A}$ & 5,0 & 6,0 \\
\hline $\mathrm{B}$ & 8,4 & 4,8 \\
\hline $\mathrm{C}$ & 8,9 & 2,4 \\
\hline
\end{tabular}

Os valores próprios pré-estabelecidos para a matriz $\mathbf{N}$, foram: $\lambda^{*}=60000$, $\lambda_{2}^{*}=50000, \lambda_{3}^{*}=40000, \lambda_{4}^{*}=30000, \lambda_{5}^{*}=20000, \lambda_{6}^{*}=10000$. 
Desta forma o valor limite para a variância de qualquer parâmetro estimado para esta rede é de $\frac{1}{10000} \mathrm{~m}^{2}=0,0001 \mathrm{~m}^{2}$, que representa o valor próprio máximo estabelecido para a matriz de covariâncias.

Os resultados da aplicação dos dois métodos na otimização do projeto da rede estão apresentados no Quadro 2.

Quadro 2 - Resultado do Processo de Otimização: Experimento 2.

\begin{tabular}{|c|c|c|c|}
\hline \multicolumn{2}{|c|}{ Método } & Quase Newton-BFGS & Newton Clássico \\
\hline \multirow{7}{*}{ Pesos } & $\mathrm{d}_{\mathrm{PA}}$ & 32827,537120012 & 22472,668290897 \\
\cline { 2 - 4 } & $\mathrm{d}_{\mathrm{PB}}$ & 24544,866942047 & 23655,685501866 \\
\cline { 2 - 4 } & $\mathrm{d}_{\mathrm{PC}}$ & 47126,907435123 & 29454,270746096 \\
\cline { 2 - 4 } & $\mathrm{d}_{\mathrm{QA}}$ & 28344,031030285 & 17917,640694476 \\
\cline { 2 - 4 } & $\mathrm{d}_{\mathrm{QB}}$ & 26208,087336893 & 26908,594769211 \\
\cline { 2 - 4 } & $\mathrm{d}_{\mathrm{QC}}$ & $\mathbf{- 9 8 8 9 , 5 9 6 9 8 3 9 3 3 2}$ & 31137,583322232 \\
\cline { 2 - 4 } & $\alpha_{\mathrm{PA}}$ & 346089,649965465 & 355469,799875962 \\
\cline { 2 - 4 } & $\alpha_{\mathrm{PB}}$ & 163852,474331488 & 344386,672321267 \\
\cline { 2 - 4 } & $\alpha_{\mathrm{PC}}$ & 316318,214151395 & 131120,442745832 \\
\cline { 2 - 4 } & $\alpha_{\mathrm{QA}}$ & 293948,206834533 & 301913,096673533 \\
\cline { 2 - 4 } & $\alpha_{\mathrm{QB}}$ & 203210,489658378 & 427116,682066829 \\
\cline { 2 - 4 } & $\alpha_{\mathrm{QC}}$ & 483592,954525169 & 199625,330405618 \\
\hline
\end{tabular}

Ambos os métodos produziram solução distinta. Este fato pode ocorrer porque o método de Newton clássico não privilegia minimizadores nem maximizadores. $\mathrm{O}$ método busca, se houver convergência, a solução mais próxima em que o gradiente se anula e este pode ser um ponto de máximo ou de mínimo ou ainda um ponto de sela. Pode ocorrer ainda que a função tenha mais que um ponto de mínimo e as respostas representem dois desses pontos distintos. A solução obtida com o algoritmo BFGS apresentou um peso negativo que, do ponto de vista da definição de peso dada em (3), não pode ser obtido na prática. Neste caso, pode-se afirmar que esta observação não coopera com a precisão final da rede planejada. Entretanto, a observação que obteve peso negativo pode ser analisada sob dois aspectos: 1) O projeto da rede apresenta número de graus de liberdade "suficiente" e pode ser descartada. Neste caso, não haverá a necessidade de ser medida em campo. 2) A observação que recebeu peso negativo não deve ser descartada. Para este caso, é suficiente aplicar um peso positivo no processo de ajustamento da rede. As demais observações devem receber pesos iguais ou superiores aos estabelecidos no algoritmo de otimização de pesos. Em termos práticos, pode-se usar o mesmo equipamento para realizar todas as observações. A precisão final da rede será atendida, desde que respeitados os critérios acima. 
Apesar das soluções apresentadas para o experimento acima serem distintas, a precisão da rede com as especificações pré-estabelecidas é garantida.

\subsection{Experimento 3}

Considera-se, nesta situação, uma rede geodésica como a representada na Figura 4, onde somente observações de distâncias são planejadas a partir de quatro pontos conhecidos com a finalidade de estimar as coordenadas do ponto A, fazendo-se uso do método dos mínimos quadrados na forma paramétrica.

Figura 4 - Rede Geodésica Bidimensional.

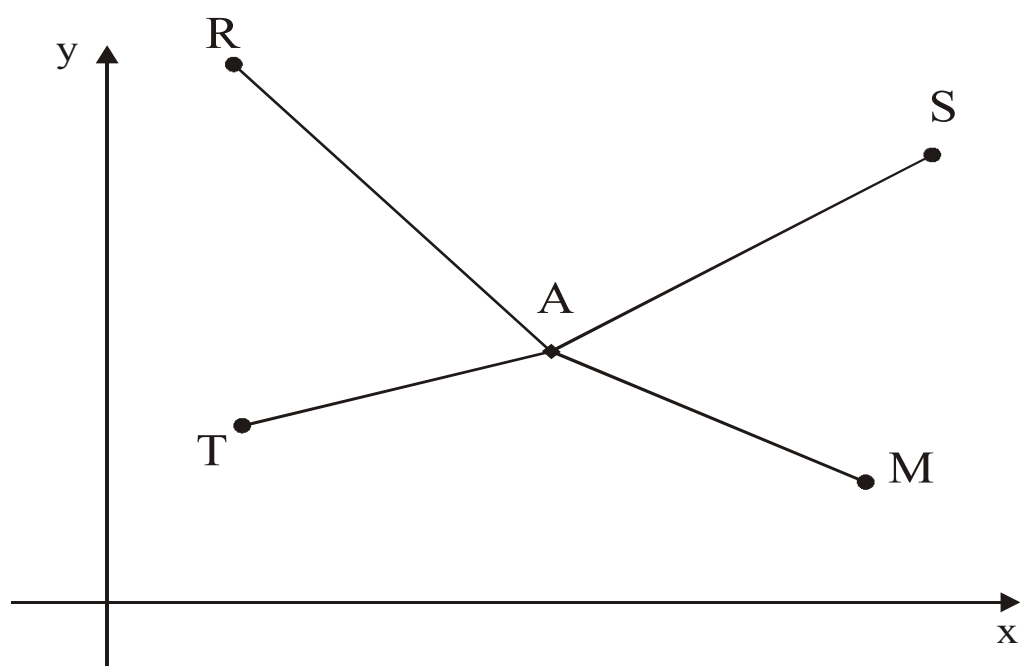

As coordenadas dos pontos conhecidos R, T, S e M e também as coordenadas aproximadas do ponto A estão apresentados na Tabela 3.

Tabela 3 - Coordenadas Fixas e Aproximadas da Rede Geodésica.

\begin{tabular}{c|c|c}
\hline \multirow{2}{*}{ Ponto } & \multicolumn{2}{|c}{ Coordenadas fixas } \\
\cline { 2 - 3 } & $\mathrm{x}$ & $\mathrm{y}$ \\
\hline $\mathrm{R}$ & 20 & 70 \\
\hline $\mathrm{T}$ & 30 & 20 \\
\hline $\mathrm{M}$ & 110 & 20 \\
\hline $\mathrm{S}$ & 120 & 60 \\
\hline Ponto & \multicolumn{2}{|c}{ Coordenadas aproximadas } \\
\hline $\mathrm{A}$ & 70 & 30 \\
\hline
\end{tabular}


Os valores próprios pré-estabelecidos para a matriz de covariâncias dos parâmetros estimados são: $\mu_{1}=0,00025$ e $\mu_{2}=0,001$. Conseqüentemente, os valores próprios utilizados no processo de otimização, ou seja, para a matriz dos coeficientes das equações normais N, são: $\lambda_{1}^{*}=\frac{1}{\mu_{1}}=4000$ e $\lambda_{2}^{*}=\frac{1}{\mu_{2}}=1000$.

Neste experimento, optou-se em inicializar o processo de otimização dos pesos em pontos distintos para cada um dos métodos descritos. O método de Newton clássico foi inicializado com os valores $\mathrm{P}^{0}=\left[\begin{array}{llll}1 & 1 & 1 & 1\end{array}\right]$ e o método

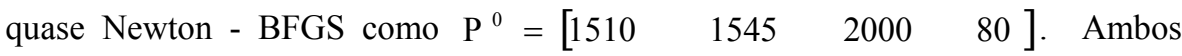
escolhidos arbitrariamente.

Os resultados da aplicação dos dois métodos, inicializados em pontos distinto, na otimização do projeto da rede estão apresentados no Quadro 3.

Quadro 3 - Resultado da Otimização para o Experimento 3

\begin{tabular}{|c|c|c|c|}
\hline \multicolumn{2}{|c|}{ Método } & Quase Newton-BFGS & Newton Clássico \\
\hline \multirow{4}{*}{ Pesos } & $\mathrm{D}_{\mathrm{RA}}$ & 1290,111667 & 1333,789388 \\
\cline { 2 - 4 } & $\mathrm{D}_{\mathrm{TA}}$ & 1623,39852 & 1191,513343 \\
\cline { 2 - 4 } & $\mathrm{D}_{\mathrm{AS}}$ & 2017,013237 & 1291,568106 \\
\cline { 2 - 4 } & $\mathrm{D}_{\mathrm{MA}}$ & 69,43523721 & 1183,129163 \\
\hline
\end{tabular}

A rede foi planejada somente observações de distâncias. Ambos os métodos propostos apresentaram solução para o planejamento escolhido. Entretanto, a solução apresentada por cada um dos algoritmos forneceu somente pesos positivos e distintos para cada método aplicado. Este fato decorre por ser o sistema não-linear, tornando possível a existência de mais de um ponto crítico. A obtenção deste ponto crítico está vinculada à aproximação do ponto de inicialização do processo, como pode ser observado no Quadro 3. Assim, as observações devem ser utilizadas no ajustamento com pesos iguais ou maiores do que os apresentados no Quadro 3. A precisão para a rede será igual ou melhor do que aquela estabelecida no seu planejamento se isto for seguido.

\section{CONCLUSÃO}

A otimização dos pesos baseada em um problema de valor próprio inverso, é uma ferramenta de grande utilidade quando se deseja projetar uma rede geodésica com especificações pré-definidas. Com base nos resultados dos experimentos, concluiu-se também que ambos os métodos apresentados tendem a produzir resultados estatisticamente confiáveis para serem aplicados a projetos de redes geodésicas bidimensionais locais.

A título de sugestão, recomenda-se que o método de Newton clássico seja primeiramente aplicado, face à sua simplicidade em relação ao método quase 
Newton - BFGS e tendo em vista que essencialmente a obtenção de um ponto de mínimo não é fundamental para o caso da otimização dos pesos das observações geodésicas. Caso o método de Newton clássico falhar na primeira tentativa, isto é, não convergir para o ponto de sela, pode-se então, usar o método quase Newton BFGS para a solução. Se ambos os métodos falharem recomenda-se reformular a matriz de configuração $\mathbf{A}$, fazendo, por exemplo, uma reavaliação em um outro ponto de aproximação para os parâmetros ou então projetando-se outras observações ou mesmo excluindo alguma das existentes.

\section{REFERÊNCIAS BIBLIOGRÁFICAS}

BRONSON, R. Matrizes. Lisboa: McGraw-Hill, 1993.

DALMOLIN, Q. Ajustamento por mínimos quadrados. $3^{a}$. ed. Curso de PósGraduação em Ciências Geodésicas, UFPR. Curitiba. 179p. 2010.

DALMOLIN, Q.; OLIVEIRA, R. O Problema de valor próprio inverso: aplicações em geodésia. Curso de Pós-Graduação em Ciências Geodésicas, UFPR. Curitiba. 169p. 2009.

DENNIS Jr., J. E.; SCHNABEL, R. B. Numerical methods for unconstrained optimization and nonlinear equations. Englewood Cliffs: Prentice-Hall. 1983.

FRIEDLAND,S.; NOCEDAL, J.; OVERTON, M. L.. The formulation and analysis of numerical methods for inverse eigenvalue problems. SIAM J. Numer. Anal. v. 24, n. 3, p. 634-667, 1987.

GEMAEL, C. Introdução ao Ajustamento de Observações: aplicações geodésicas. Editora da UFPR. Curitiba, 1994.

GRAFAREND, E. W.; SANSO F. Optimization and design of geodetic networks. Spring Verlag, Berlin-Heidelberg-New York-Tokio, 1985.

JÄGER, R.; KALTENBACH, H. Spectral analysis and optimization of geodetic networks based on eigenvalues and aigenfunctions. Manuscripta geodaetica, Berlin, v. 15, n. 5, p.302-311, 1990

MARTINEZ, J. M.; SANTOS, S. A. Métodos computacionais de otimização. In: Colóquio Brasileiro de Matemática, 20, Rio de Janeiro: Instituto de Matemática Pura e Aplicada. 24-28 julho, 256 p., 1995.

NINKOV, T.; SCHMITT, G. Eine Methode Gewichtsoptimierung in geodätischen Netzen. Allgemeine Vermessungs-Nachrichten, Karlsruhe, v. 90, n. 6, p. 216222, 1983.

NOCEDAL, J.; WRIGHT, S.T. Numerical optimization. Springer series in operations research. Springer-Verlag New York. 635 p., 1999.

OLIVEIRA, R. Otimização dos pesos das observações geodésicas pelo problema de valor próprio inverso. Curitiba. Dissertação de Mestrado. Curso de PósGraduação em Ciências Geodésicas da Universidade Federal do Paraná, 95 p., 2003.

WANG, S.G. \& CHOW, S. C. Advanced Linear Models: Theory and Applications. Dekker, New York, 1994. 


\section{Outras bibliografias consultadas}

BIEGLER-KÖNIG, F. W. (1981). A Newton iteration process for inverse eigenvalue problems. Numerische Mathematik, n. 37, p. 349-354.

DEREN, L.; YIONGQIAN, Z. (1991). Optimization and design of geodetic networks in consideration of accuracy and reliability. Allgemeine VermessungsNachrichten, Karlsruhe, v. 91, n. 8, p. 27-33.

RIEDLAND, S.; NOCEDAL, J.; OVERTON, M. L. (1987). The formulation and analysis of numerical methods for inverse eigenvalue problems. Siam $J$. Numerical Anal., v. 24, n. 3, p. 635-667.

GRAFAREND, E. (1974). Optimization of geodetic networks. Bolletino di Geodesia e Scienze Affini, Firenze, v. 33, n. 4, p. 351-406.

(Recebido em janeiro de 2010. Aceito em setembro de 2010.) 